\title{
Prevalence, Patterns, and Cost of Care for Children with Cerebral Palsy Enrolled in Medicaid Managed Care
}

\author{
Sonia Pulgar, MPH; Savreet Bains, MS; Judith Gooch, MD; Henry Chambers, MD; \\ Garey H. Noritz, MD; Edward Wright, MD; Tia Goss Sawhney, DrPH, FSA, MAAA; \\ Bruce Pyenson, FSA, MAAA; and Christine Ferro, CHFP
}

\begin{abstract}
BACKGROUND: In the United States, many children with cerebral palsy (CP) obtain health care coverage through managed Medicaid, but little is known about the current demographics or management of this high-need, complex population.
\end{abstract}

OBJECTIVE: To develop U.S. population-level information about the prevalence of $\mathrm{CP}$, management patterns, and costs.

METHODS: Data (2013-2015) were analyzed from a managed Medicaid database with coverage of children and adolescents in 15 states. Analyses included demographic information and use of 10 prespecified CP management options often used to manage spasticity. Code-based algorithms were applied to indicate presence of spasticity and determine the likely ambulatory status.

RESULTS: In this claims analysis, the prevalence estimate of CP was 1.78 per 1,000 patients. Most $(69.8 \%)$ children with CP had spasticity, of which $20.8 \%$ had hemiplegia, $15.6 \%$ diplegia, $32.9 \%$ quadriplegia, and $30.5 \% \mathrm{CP}$ unspecified. Overall, $42.4 \%$ of children with $\mathrm{CP}$ were not treated with any of the $10 \mathrm{CP}$ management options via Medicaid. Among treated children, the most common management options were physical therapy (37.1\%), orthotics $(29.9 \%)$, oral baclofen (13.5\%) and botulinum toxins $(9.4 \%)$. Overall annualized Medicaid costs were higher for children with $\mathrm{CP}$ versus children in the overall database population $(\$ 22,383$ vs. $\$ 1,358)$. Within the CP population, costs were higher for those children who were likely nonambulatory than for those who were likely ambulatory $(\$ 43,687$ vs. $\$ 10,368$, respectively).

CONCLUSIONS: Most children with CP have spasticity, and the costs of care are high. This study highlights wide variation in the way CP is managed, with many young patients not receiving $\mathrm{CP}$ management options via Medicaid.

J Manag Care Spec Pharm. 2019;25(7):817-22

Copyright @ 2019, Academy of Managed Care Pharmacy. All rights reserved.

\section{What is already known about this subject}

Cerebral palsy (CP) is the most common cause of physical disability in childhood.

- Therapeutic management is necessary to promote childhood development, with a wide range of treatment options ranging from physical and occupational therapy through pharmacologic treatments to neurosurgical or orthopedic surgery.

\section{What this study adds}

This study found that about $70 \%$ of children with CP covered by managed Medicaid have spasticity.

For children with $\mathrm{CP}$, average annualized Medicaid costs were $\$ 22,383$ in 2013-2015.

Many Medicaid-eligible children with CP (42.4\%) in the data source did not receive therapies commonly used for CP management via Medicaid.

( erebral palsy (CP) is a group of neurologic disorders typically caused by a nonprogressive lesion or abnormality of the developing brain that appears in infancy or early childhood and permanently affects body movement, muscle coordination, and balance. ${ }^{1,2}$ The etiologic events typically occur in an early phase of fetal and infant cerebral development and have been reported to affect between 1.5 and $>4$ of every 1,000 live births worldwide. ${ }^{3}$ In addition, some children develop CP as the result of brain damage (e.g., due to meningoencephalitis or traumatic brain injury) in the first few months or years of life. ${ }^{4}$

The clinical presentation of CP depends on the size and location of the brain abnormality, and as such, children can show wide variation in the type of movement disorder, body parts affected, and effect on function. ${ }^{4}$ Several ways of classifying this diverse disorder have been proposed. ${ }^{1}$ According to Task Force on Childhood Motor Disorders criteria, ${ }^{5}$ most children with CP ( 77\%) have spasticity as their predominant motor symptom, ${ }^{3}$ although many display components of spasticity and dystonia. ${ }^{1}$ Spastic forms of CP are further broken down into monoplegia (affecting 1 limb), hemiplegia (affecting 1 side of the body), diplegia (affecting both limbs, either arms or legs), and tetraplegia/quadriplegia (affecting all 4 limbs). ${ }^{4,6}$ Given that CP often starts at birth and is a lifelong disorder, there is consensus that the disorder needs to be considered in the context of ongoing childhood development, functioning, and family impact. ${ }^{4}$ Therapeutic management is frequently necessary to promote childhood development, with options ranging from conservative treatment (e.g., physical and occupational therapy) to pharmacologic treatments (e.g., oral antispasticity medications and injected botulinum toxins) and neurosurgical or orthopedic surgery. 
In the United States, many children with CP obtain health care coverage through managed Medicaid, ${ }^{7}$ but little is known about the current demographics or management of this highneed, complex population. Medicaid managed care provides delivery of health benefits and services through contracted agreements between state Medicaid plans and managed care organizations. These contracts are intended to reduce Medicaid program costs and better manage utilization of health services. The aim of this analysis was to investigate Medicaid managed care administrative claims data in order to develop populationlevel information about the prevalence of $\mathrm{CP}$ and its management patterns and costs.

\section{Methods}

\section{Population}

Data were retrospectively analyzed from a proprietary database of managed Medicaid (Milliman Managed Medicaid Database, Seattle, WA) with coverage from 15 states, encompassing an average annual enrollment of 5.3 million individuals throughout 2013-2015.

Patients aged 2-20 years were identified, according to Medicaid's consideration of disabled patients aged $<21$ years to be eligible children, regardless of continuity of enrollment. Eligibility for the analysis was dependent on having $\geq 11$ months of medical and pharmacy coverage in a calendar year. Children with CP were identified using International Classification of Diseases, Ninth Revision, Clinical Modification (ICD-9-CM; infantile cerebral palsy 343.x and athetoid cerebral palsy 333.71) or International Classification of Diseases, Tenth Revision, Clinical Modification (ICD-10-CM) diagnostic codes (cerebral palsy G80.x) and were eligible for inclusion in the analysis if they had a CP diagnosis code in any position on $\geq 1$ inpatient or observation claim or on $\geq 2$ emergency department, nonacute inpatient, or outpatient claims occurring on 2 distinct dates of service over the 3-year period, including years with $<11$ months.

Diagnosis codes in the data indicated the type of $\mathrm{CP}$ (spastic, ataxic/athetoid, and CP not otherwise specified) and anatomic topography (monoplegia, hemiplegia, diplegia, and triplegia/quadriplegia). When codes conflicted, a hierarchy was applied: quadriplegia $>$ hemiplegia $>$ diplegia $>$ monoplegia $>$ not specified "plegia." Type of CP and anatomic topography were assigned once for the 3-year period. Management option use was identified using medical and pharmaceutical claims. Claims per patient-year (patient-year defined as a calendar year with $\geq 11$ months of enrollment) for the following common $\mathrm{CP}$ therapies used for CP management were analyzed: physical therapy; orthotics; orthopedic surgery; rhizotomies; casting; baclofen (injectable, pump, and oral forms); diazepam; botulinum toxin (BoNT); and antispasm medications. These therapies were selected because they are often used for spasticity management.

\section{Data Analyzed}

Claimant demographics, management option use, and medical equipment and supplies used were assessed per patient-year. Service costs included all claims paid by the Medicaid managed care plan, including hospitalizations, outpatient office visits, home nursing, medications, durable medical equipment, medical supplies, home health, and long-term care.

Children with any CP diagnosis codes over the 3-year period indicative of spasticity were assumed to have spasticity, as were children with diagnosis and/or procedure codes indicative of joint contractures, muscle contractures, hip dislocation, spasms, and hypertonia. In addition, a coding algorithm was developed to assign a child's likely ambulatory status (likely nonambulatory, likely ambulatory, and unknown). A likely nonambulatory status was assigned to children with a tetraplegia/quadriplegia diagnosis over the 3-year period. Children with monoplegia and hemiplegia were assigned a likely ambulatory status. Children with diplegia and unspecified CP were assigned as likely nonambulatory status, if they had costs for use of powered wheelchairs and related supplies, lift positioning devices, orthopedic surgeries indicative of nonambulation, or if they used intrathecal drugs. Children were assigned a likely ambulatory status if they had a diagnosis of impaired gait and used ambulatory orthotics and/or walking assistance devices such as walkers, crutches, and canes. Some children were unassignable and were labeled as unknown ambulatory status.

A total of 7,158 child-years were identified for the CP population. Each child-year was analyzed separately and flagged for each category. A patient who was included in the analysis was included for 1-3 years (2013-2015) with management pattern and costs for each year.

\section{Results}

\section{Description of the Medicaid CP Population}

Of the 2,154,479 children with $\geq 1$ patient-year identified in the database, 3,294 children aged 2-20 years (with a total of 7,158 child-years) were identified as having CP and were included in the $\mathrm{CP}$ analyses. This selection produced a prevalence estimate of 1.78 per 1,000 (7,158 CP child-years/4 million total child-years) of the child Medicaid population (Table 1). The prevalence rate was higher in males. Prevalence estimates by geography suggested potential regional variability; however, this may have been influenced by the source data coding and/or limitation to 15 anonymous states, which could not be explored further (Table 1).

Most (69.8\%) children had coding indications of spasticity, and $4.4 \%$ had a primarily athetoid/ataxic form of CP. However, a quarter $(25.7 \%)$ of the records did not specify the predominant type of CP. As analyzed by child-years, $69.5 \%$ of children with CP had their topography of affected limbs described. Of these, 20.8\% had hemiplegia; 15.6\% had diplegia; 32.9\% had 


\begin{tabular}{|c|c|}
\hline $\begin{array}{ll}\text { TABLE 1 Prev } \\
\end{array}$ & $\begin{array}{l}\text { per } 1,000 \text { of the } \\
\text { ion }\end{array}$ \\
\hline Category & Prevalence per 1,000 \\
\hline Overall (aged 2-20 years) & 1.78 \\
\hline Sex & \\
\hline Male (aged 2-20 years) & 2.00 \\
\hline Female (aged 2-20 years) & 1.55 \\
\hline Age, years & \\
\hline $2-3$ & 1.52 \\
\hline $4-8$ & 1.59 \\
\hline $9-13$ & 1.68 \\
\hline $14-18$ & 1.93 \\
\hline $19-20^{\mathrm{a}}$ & 4.05 \\
\hline Region & \\
\hline Northwest & 1.92 \\
\hline North Central & 2.05 \\
\hline Northeast & 2.36 \\
\hline South Central & 1.23 \\
\hline Southeast & 0.90 \\
\hline Southwest & 1.89 \\
\hline $\begin{array}{l}\text { In non-ACA expansion states } \\
\text { age } 19 \text { years, unless they have } \\
19-20 \text { years is disproportiona } \\
\text { ACA = Affordable Care Act; } C\end{array}$ & $\begin{array}{l}\text { en leave Medicaid upon th } \\
\text {; therefore, the population }\end{array}$ \\
\hline
\end{tabular}

quadriplegia; $0.1 \%$ had monoplegia; and the remaining claims had unspecified CP.

Based on the coding algorithms applied, approximately one third $(30.5 \%)$ of children was estimated as likely ambulatory, and another third (33.8\%) was estimated as likely nonambulatory. Due to lack of ambulatory indicative data, it was not possible to assign ambulatory status for the remaining third (35.7\%).

\section{Use of Therapies for CP Management}

Overall, $57.6 \%$ of children with CP used at least 1 of the previously mentioned common CP therapies for CP management over the course of a year. Nearly half (42.4\%) of children with $\mathrm{CP}$ did not appear to use these common options via Medicaid (as measured by paid claims). The most common management options were physical therapy (37.1\%), orthotics (29.9\%), and BoNT injections (13.5\%) measured as the proportion of childyears in which the treatment option appeared in the database (Figure 1).

$\mathrm{CP}$ management option use rates varied based on likely ambulatory status. Whereas the use of physical therapy and orthotics was similar between the 2 groups, likely nonambulatory children were more than 5 times as likely to use oral antispasticity medications (18.4\% vs. $3.5 \%$ ) than likely ambulatory children.

For any single year, almost 1 in 10 (9.4\%) children with CP were treated with BoNT, and its use was higher in likely nonambulatory children versus likely ambulatory children (16.9\% vs. $10.4 \%)$ and much higher than in children whose ambulatory status was unknown (1.5\%). These differences were related to the types of CP treated with BoNT. Overall, $60 \%$ of child-years for BoNT use was for children with quadriplegia, and 25\% was for children with hemiplegia. Age was another key factor driving BoNT use-children aged 4-8 years were most likely to be treated with a BoNT (13.2\%), and older children aged 14-18 years and 19-20 years were the least likely to be treated with a BoNT (6.9\% and 3.7\%, respectively).

\section{Annual Costs of CP Management}

Average annualized Medicaid costs were more than 15 times higher for children with CP compared with the overall Medicaid population $(\$ 22,383$ for children with CP vs. $\$ 1,358$ for children in the Medicaid population). For children with CP, costs were mostly related to medical services (59.8\%) and home health/long-term care/hospice care (29.1\%). Pharmacy costs accounted for $11.1 \%$ of spend.

Costs were higher in children who were likely nonambulatory than in children who were likely ambulatory ( $\$ 43,687$ vs. $\$ 10,368$, respectively). Incontinence services were the most used service in likely nonambulatory children (63.3\% of childyears), and physical therapy was the most used service in likely ambulatory children ( $42.8 \%$ of child-years). The largest overall contributors to spend were home health, long-term care, and/or hospice for likely nonambulatory children $(\$ 14,528$ per patientyear) and inpatient hospital stays for likely ambulatory children (\$2,096 per patient-year).

\section{Discussion}

Our analysis highlights the seriousness of CP. The estimated prevalence of CP in our analysis was 1.78 per 1,000 children, which is not rare and is on the lower end of published reports where prevalence estimates range from 1.5 to more than 3 per 1,000 children, although these are also limited to the specific geographies used in our analysis. ${ }^{8-12} \mathrm{~A}$ lower prevalence may be explained in part by the exemption of some disabled children from mandatory managed care enrollment in Medicaid plans. Such exemptions often have the result of skewing the population toward the less disabled, although we noted that children with quadriplegia (often the most severe form of CP) accounted for about $33 \%$ of our population.

The predominance of spasticity as a key presenting motor symptom (reported in 70\% of children with CP) is lower than previous reports that have estimated that spasticity affects as many as $89 \%$ of U.S. children with CP. ${ }^{11}$ This number could be lower because spasticity is not recorded as a diagnosis on medical claims. For the purposes of this analysis, we assumed CP patients with a hemiplegia, diplegia, or quadriplegia indication (as opposed to those without a specific diagnosis of $\mathrm{CP}$ subtype) had spasticity. 


\section{FIGURE 1 Utilization of Key CP Management Therapies, Overall and by Likely Ambulatory Status} ( $N=7,158$ Child-Years)

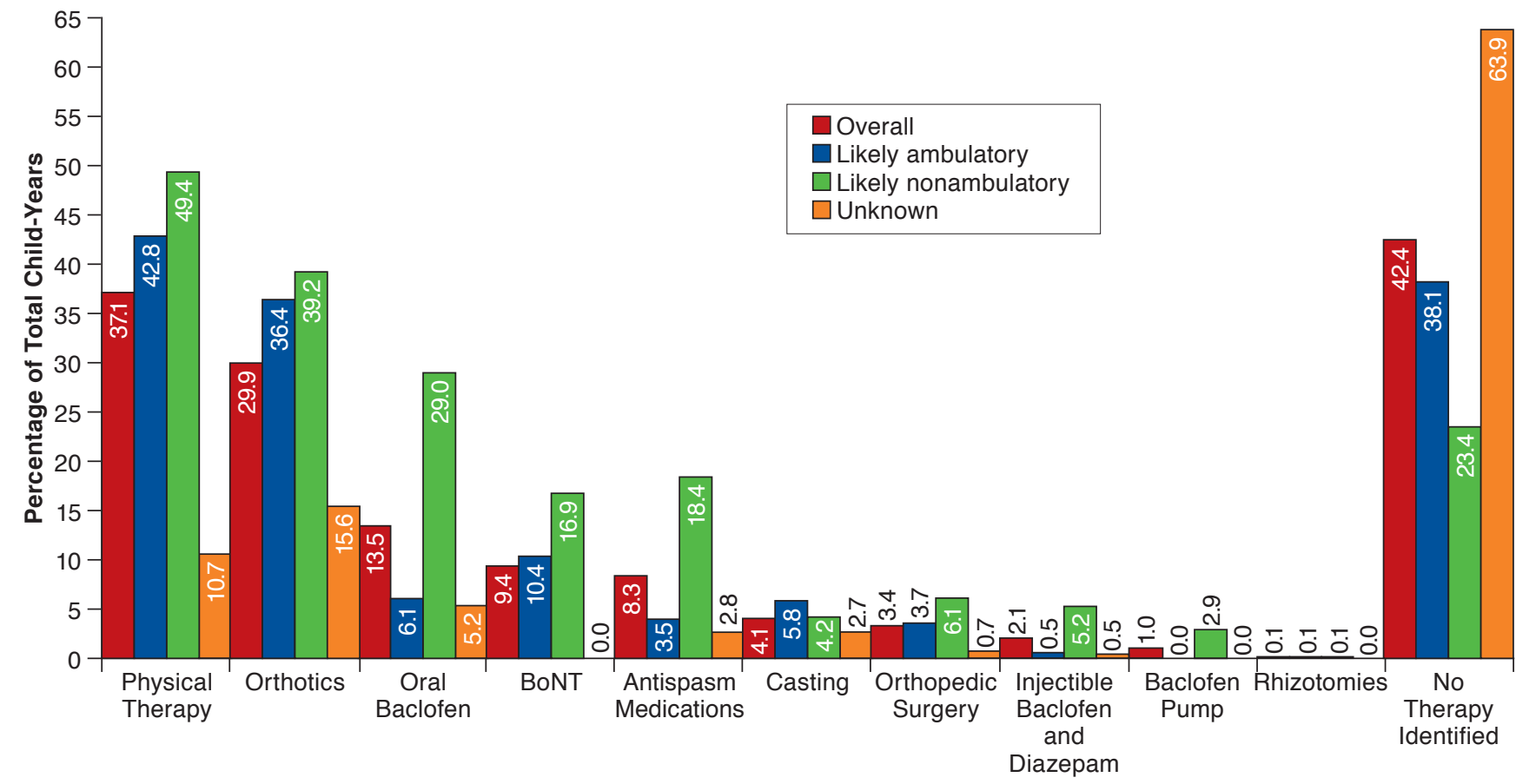

BoNT = botulinum toxin; $C P=$ cerebral palsy.

While CP is considered nonprogressive in terms of the original brain lesion, the presence of spasticity and other features of the upper motor neuron syndrome often causes secondary progressive musculoskeletal problems that develop during the rapid growth of childhood. ${ }^{13,14}$ Musculoskeletal problems such as tissue shortening and contractures can worsen over time, so it is important to recognize and manage spasticity because without proper management secondary events will continue into adulthood..$^{15}$ In this respect, the dystonia characterized by repetitive movements and abnormalities in posture is challenging to identify in claims data and was not included in this analysis because diagnosis codes do not accurately distinguish between symptoms of spasticity and dystonia.

Another consideration is the often confusing nomenclature of $\mathrm{CP}$, where "dystonic $\mathrm{CP}$ " is often considered part of the "extrapyramidal" CP type, which includes the athetoid/ataxic forms of CP. ${ }^{2}$ In the absence of clear terminology and coding, physicians may hesitate to record symptoms of limb dystonia when spasticity is also present, leading to higher than expected rates of unspecified CP (26\% in our study vs. $14 \%$ in the literature $^{16}$ ) and lower rates of spasticity.

Mobility is such an important consideration in $\mathrm{CP}$ that many clinicians prefer to use the Gross Motor Function Classification System (GMFCS, which focuses on walking ability) when choosing the surgeries, treatments, therapies, and assistive technology most likely to result in the best outcome for the individual child. ${ }^{17}$ The GMFCS is not used in medical claims coding, so walking ability is not accurately coded. For this reason, we developed a novel algorithm to estimate the likely ambulatory status of each child. Since we could not estimate the level of ambulation (e.g., outdoors ambulation vs. indoor only), the algorithm is a relatively blunt instrument that is limited by the lack of exact data. Nevertheless, our findings do highlight the significant effect that the child's ambulatory status has on the cost of care. We were unable to estimate the ambulatory status of just over one third of the children, often because of the absence of diagnosis and procedure codes indicative of ambulation or nonambulation. This subset of children requires further exploration to better understand their clinical presentation and management.

Consensus recommendations are for most children with $\mathrm{CP}$ to be actively treated by a multidisciplinary team. ${ }^{4,18,19}$ Our observation that no Medicaid treatment was recorded for up to $42.4 \%$ of children with CP (and increasing to $63.9 \%$ of children with unknown ambulatory status) is of some concern. It is possible that these children have milder CP that does not require management or that they access less common management options or management options not covered under the 
Medicaid system. Nonetheless, higher rates of treatment might be expected.

Although physical therapy is considered to be the cornerstone of CP management, ${ }^{4,18}$ we found evidence of physical therapy in less than half of all child enrollment exposure years (child-years). Future analyses might investigate the use of management options by age groups; for example, the use of physical therapy may dissipate after the early elementary years. ${ }^{20}$

Since extent of ambulation can be considered roughly negatively correlated with severity, it is not surprising that the children considered to be likely nonambulatory had higher management option use, higher rates of care, and higher cost of care compared with those considered likely ambulatory. For example, utilization rates of antispasticity medications, including all forms of baclofen, were higher in nonambulatory children. Costs of hospitalizations and specialized home health/ long-term care were also key drivers for the total medical spend for these more severely affected children.

Of note, the rate of BoNT injections was higher in children considered likely nonambulatory (17\% vs. $10 \%)$ and was higher in younger versus older children. The use of BoNT in likely nonambulatory children may suggest that chemodenervation with BoNT could be used as palliation to improve ease of care, pain, posture, and other problems. However, the most robust level of evidence for the efficacy of BoNT is now in ambulatory children with lower limb spasticity in whom the aim of treatment is often to improve gait. ${ }^{21-23}$ In this respect, the higher use of BoNT in younger age groups could, in part, reflect the frequent goal of improving gait patterns in ambulatory patients before they become fixed around the age of 6-8 years and delaying the need for orthopedic surgery for as long as possible. ${ }^{14}$ It should be noted that the first approval for a BoNT in pediatric spasticity by the U.S. Food and Drug Administration occurred after the cut-off dates for this analysis-abobotulinumtoxinA (Dysport, Ipsen Pharma, Wrexham, UK) was approved for the treatment of lower limb spasticity in pediatric patients aged $\geq 2$ years in July 2016 .

\section{Limitations}

This study has some limitations to consider. These data may not be representative of the entire United States, since they rely on Medicaid claims from a subset of managed care organizations in 15 states, and not all children with CP covered by Medicaid are enrolled in managed care; however, this is one of the largest samples of patients aged $<21$ years with $\mathrm{CP}$. These data are gathered from adjudicated claims that may be incomplete or inaccurate. For example, claims diagnosis codes may not include all the diagnoses present for each patient, such as codes indicative of spasticity. The identification of affected limb topography, spasticity, and ambulatory status used independently developed algorithms that have not been validated. In addition, there are substantial variations in state Medicaid policies, managed care benefits, and payment policies. U.S patient registries that can follow children with CP (regardless of type of health coverage) through their daily care could address these issues. Such registries already exist in other developed countries ${ }^{24,25}$ and provide a snapshot of current care and offer insights into how patterns of care for children with $\mathrm{CP}$ progress over time.

\section{Conclusions}

To the best of our knowledge, this is one of the largest analyses of how children with CP are treated in the U.S. Medicaid managed health care system. CP prevalence was notable in this population, and most children with CP were affected by spasticity. The cost of care was high, reflecting the multidisciplinary approach required for long-term CP care. Most concerning, however, was the appearance of a large proportion of children with CP covered by Medicaid managed care who did not receive any treatment for physical disability.

\section{Authors}

SONIA PULGAR, MPH, and SAVREET BAINS, MS, Ipsen Biopharmaceuticals, Basking Ridge, New Jersey. JUDITH GOOCH, MD, Utah Neuro Rehabilitation, Murray, Utah; HENRY CHAMBERS, MD, Rady Children's Hospital-San Diego, San Diego, California; GAREY H. NORITZ, MD, Nationwide Children's Hospital, Columbus, Ohio; and EDWARD WRIGHT, MD, Texas Children's Hospital, The Woodlands, Texas. TIA GOSS SAWHNEY, DrPH, FSA, MAAA; BRUCE PYENSON, FSA, MAAA; and CHRISTINE FERRO, CHFP, Milliman, New York, New York.

AUTHOR CORRESPONDENCE: Sonia Pulgar, MPH

E-mail: sjapulgar@gmail.com.

\section{DISCLOSURES}

This analysis was funded by Ipsen Biopharmaceuticals and conducted by Milliman. Pulgar and Bains were employees of Ipsen Biopharmaceuticals during the conduct of this study. Chambers is a consultant for OrthoPediatrics and an employee of the University of California. Pyenson and Ferro are employees of Milliman, as was Sawhney during the analysis. Gooch, Noritz, and Wright report no conflicts of interest.

Part of this work was presented as a poster at TOXINS 2017: Basic Science and Clinical Aspects of Botulinum and Other Neurotoxins, held January 18-21, 2017, in Madrid, Spain.

\section{ACKNOWLEDGMENTS}

The authors thank Anita Chadha-Patel, PhD (ACP Clinical Communications, funded by Ipsen Pharma) for editorial support (literature searching, editing, and final styling of the paper for submission) in the development of this manuscript. 


\section{REFERENCES}

1. Christine C, Dolk H, Platt MJ, et al. Recommendations from the SCPE collaborative group for defining and classifying cerebral palsy. Dev Med Child Neurol Suppl. 2007;109:35-38

2. Bax M, Goldstein M, Rosenbaum P, et al. Proposed definition and classification of cerebral palsy, April 2005. Dev Med Child Neurol. 2005;47(8):571-76.

3. Centers for Disease Control and Prevention. Data and statistics for cerebral palsy. Updated March 9, 2018. Available at: http://www.cdc.gov/ ncbddd/cp/data.html. Accessed May 9, 2019.

4. Graham HK, Rosenbaum P, Paneth N, et al. Cerebral palsy. Nat Rev Dis Primers. 2016;2:15082.

5. Sanger TD, Delgado MR, Gaebler-Spira D, Hallett M, Mink JW, Task Force on Childhood Motor D. Classification and definition of disorders causing hypertonia in childhood. Pediatrics. 2003;111(1):e89-97.

6. Kuban KC, Allred EN, O'Shea M, et al. An algorithm for identifying and classifying cerebral palsy in young children. J Pediatr. 2008;153(4):466-72.

7. Centers for Medicare $\&$ Medicaid Services. Medicaid managed care enrollment and program characteristics, 2014. Spring 2016. Available at: https:// www.medicaid.gov/medicaid-chip-program-information/by-topics/data-andsystems/medicaid-managed-care/downloads/2014-medicaid-managed-careenrollment-report.pdf. Accessed May 9, 2019.

8. Haerer AF, Anderson DW, Schoenberg BS. Prevalence of cerebral palsy in the biracial population of Copiah County, Mississippi. Dev Med Child Neurol. 1984;26(2):195-99.

9. Yeargin-Allsopp M, Van Naarden Braun K, Doernberg NS, Benedict RE, Kirby RS, Durkin MS. Prevalence of cerebral palsy in 8-year-old children in three areas of the United States in 2002: a multisite collaboration. Pediatrics. 2008;121(3):547-54.

10. Paneth N, Hong T, Korzeniewski S. The descriptive epidemiology of cerebral palsy. Clin Perinatol. 2006;33(2):251-67.

11. Arneson CL, Durkin MS, Benedict RE, et al. Prevalence of cerebral palsy: Autism and Developmental Disabilities Monitoring Network, three sites, United States, 2004. Disabil Health J. 2009;2(1):45-48.

12. Van Naarden Braun K, Doernberg N, Schieve L, Christensen D, Goodman A, Yeargin-Allsopp M. Birth prevalence of cerebral palsy: a population-based study. Pediatrics. 2016;137(1):1-8.

13. Simpson DM, Gracies JM, Graham HK, et al. Assessment: botulinum neurotoxin for the treatment of spasticity (an evidence-based review): report of the Therapeutics and Technology Assessment Subcommittee of the American Academy of Neurology. Neurology. 2008;70(19):1691-98.
14. Molenaers G, Van Campenhout A, Fagard K, De Cat J, Desloovere K. The use of botulinum toxin A in children with cerebral palsy, with a focus on the lower limb. J Child Orthop. 2010;4(3):183-95.

15. Morgan P, McGinley J. Gait function and decline in adults with cerebral palsy: a systematic review. Disabil Rehabil. 2014;36(1):1-9.

16. Christensen D, Van Naarden Braun K, Doernberg NS, et al. Prevalence of cerebral palsy, co-occurring autism spectrum disorders, and motor functioning-Autism and Developmental Disabilities Monitoring Network, USA, 2008. Dev Med Child Neurol. 2014;56(1):59-65.

17. Palisano R, Rosenbaum P, Walter S, Russell D, Wood E, Galuppi B. Development and reliability of a system to classify gross motor function in children with cerebral palsy. Dev Med Child Neurol. 1997;39(4):214-23.

18. Strobl W, Theologis T, Brunner R, et al. Best clinical practice in botulinum toxin treatment for children with cerebral palsy. Toxins. 2015;7(5):1629-48.

19. Delgado MR, Hirtz D, Aisen M, et al. Practice parameter: pharmacologic treatment of spasticity in children and adolescents with cerebral palsy (an evidence-based review): report of the Quality Standards Subcommittee of the American Academy of Neurology and the Practice Committee of the Child Neurology Society. Neurology. 2010;74(4):336-43.

20. Kim DA, Hong HS, Lee HY, Lee HS, Kang MS. Age specificity in general and rehabilitation medical services in children with cerebral palsy. Ann Rehabil Med. 2014:38(6):784-90.

21. Delgado MR, Tilton A, Russman B, et al. AbobotulinumtoxinA for equinus foot deformity in cerebral palsy: a randomized controlled trial. Pediatrics. 2016;137(2):e20152830

22. Tilton A, Russman B, Aydin R, et al. AbobotulinumtoxinA (Dysport ${ }^{\circledR}$ ) improves function according to goal attainment in children with dynamic equinus due to cerebral palsy. J Child Neurol. 2017;32(5):482-87.

23. Delgado MR, Bonikowski M, Carranza J, et al. Safety and efficacy of repeat open-label abobotulinumtoxinA treatment in pediatric cerebral palsy. J Child Neurol. 2017; 32(13):1058-64

24. Oskoui M, Joseph L, Dagenais L, Shevell M. Prevalence of cerebral palsy in Quebec: alternative approaches. Neuroepidemiology. 2013;40(4):264-68.

25. Surveillance of Cerebral Palsy in Europe. Surveillance of cerebral palsy in Europe: a collaboration of cerebral palsy surveys and registers. Surveillance of Cerebral Palsy in Europe (SCPE). Dev Med Child Neurol. 2000;42(12):816-24 